\title{
Laccase activation in deep eutectic solvents
}

Mariah L. Toledo ${ }^{\dagger, t}$, Matheus M. Pereirat, Mara G. Freire ${ }^{\dagger}$, João P. A. Silva $a^{\dot{t}}$, João A. P. Coutinho $^{\dagger}$, Ana P. M. Tavares t* $^{*}$

${ }^{\dagger}$ CICECO - Aveiro Institute of Materials, Chemistry Department, University of Aveiro, Campus Universitário de Santiago, 3810-193 Aveiro, Portugal

†Engineering School of Lorena, Chemical Engineering Department, University of São Paulo, Estrada municipal do Campinho, S/N, Campinho, 12602-810, Lorena, São Paulo, Brazil

*Corresponding author: aptavares@ua.pt

KEYWORDS: Deep eutectic solvents, enzyme activity, green solvents, laccase, molecular docking 


\section{ABSTRACT}

The research on alternative solvents and co-solvents is a relevant aspect when envisioning the improvement of biocatalytic reactions. Among these solvents and co-solvents, deep eutectic solvents (DES) may be considered as customizable new reaction media for biocatalysis. Accordingly, in this work, sixteen DES aqueous solutions, as well as of the individual DES components at the same conditions have been investigated in laccasecatalyzed reactions. Cholinium- and betaine-based DES formed with polyols at different molar ratio and concentrations were evaluated. The results reported show that in presence of most DES the laccase activity is preserved and, with a particular DES, enhanced up to 200\%. Molecular docking studies demonstrated that while most DES components establish hydrogen-bonds with the enzyme amino acids, those that establish stronger interactions with the enzyme (expressed by absolute values of docking affinity energies) lead to an enhanced laccase activity. Finally, the laccase stability was evaluated in additional tests under extreme storage temperatures $\left(60^{\circ} \mathrm{C}\right.$ and $\left.-80^{\circ} \mathrm{C}\right)$. Although no significant protection to high temperatures was afforded by DES, an enhanced laccase activity when stored at low temperatures was found, at least up to 20 days. Combining experimental results and molecular docking this work shows that DES can be designed as co-solvents to improve biocatalytic reactions. 


\section{INTRODUCTION}

The research on alternative and greener solvents has led to advances in biocatalysis by allowing to perform enzymatic bioreactions in partially or fully nonaqueous environments. ${ }^{1,2}$ This fact is of particular relevance when dealing with hydrophobic substrates, where the presence of organic solvents could promote enzyme deactivation or denaturation. ${ }^{3}$ During the last decades, promising alternative solvents, such as ionic liquids (ILs), have been reported as solvents or co-solvents in enzyme-catalysed reactions with lipase, laccase, peroxidase, among others. ${ }^{4}$ However, some ILs based on pyridinium or imidazolium cations, most of the times combined with fluorinated anions, may raise some toxicity and biodegradability concerns ${ }^{5}$ and may lead to enzymes deactivation. ${ }^{6}$ In addition to ILs as alternative solvents and bioreaction media, deep eutectic solvents (DES) have been attracting widespread interest from academics and industrial sector since these solvents can be produced by the simple mixing of low-cost and safe compounds, most of the times from natural sources. ${ }^{7}$ Contrarily to ILs that are pure compounds composed solely of anions and cations, DES are a mixture of weak Lewis acids (hydrogen bond donor (HBD)) and bases (hydrogen bond acceptor (HBA)) which may contain anionic and/or cationic species (e.g. ammonium or phosphonium salts) mixed with non-ionic species. This means that although some physical properties of DES may be similar to ILs, their chemical properties are different. DES have the same 'designer solvent' characteristic of ILs, but with a much broader possibility for design since the solvent properties can be additionally fine-tuned by selecting different DES components at different proportions. ${ }^{8}$ In biotransformations, DES can be used as solvents, cosolvents or as a second phase in water-DES mixtures. ${ }^{9}$ 
Laccases are multicopper oxidases catalysing oxidative reactions. This type of enzymes only uses molecular oxygen and the substrate to initiate catalysis, i.e., electrons are removed from the reducing substrate molecules and transferred to oxygen to produce water. ${ }^{10}$ Laccase has received great attention from both academia and industry due to these simple requirements and ability to degrade a diversity of substrates of industrial interest. As reviewed in the literature, ${ }^{11,12}$ laccase oxidizes an extensive variety of organic and inorganic compounds, being applied in the bioremediation of phenolic compounds and dyes, and in pulp and paper processing, biosensors, medical care and synthesis of fine chemicals. However, some of these laccase substrates are highly hydrophobic and cannot be completely dissolved in aqueous medium, requiring therefore the use of organic solvents. ${ }^{13}$ Thus, DES may be considered potentially safer alternatives to organic solvents in enzymatic reactions involving laccases. ${ }^{14}$

The use of DES prevents enzymes denaturation and deactivation often detected in conventional organic solvents, as observed in some works with horseradish peroxidase ${ }^{8}$ and hydrolases. ${ }^{15}$ To date, only two works on DES as alternative solvents for laccasecatalyzed reactions has been reported. ${ }^{16,17}$ Khodaverdiana et al. ${ }^{16}$ show encouraging results for laccase activity and stability using betaine-based natural DES. Amongst the DES studied, laccase was found to be more active and stable in the glycerol:betaine DES at a molar ratio of 2:1. Additionally, the authors proved that laccase was more stable and efficient in a glycerol:betaine DES than in aqueous solutions comprising the DES components. ${ }^{16}$ Ünlü et al., studied the polycatechin synthesis by laccase in DES composed of choline chloride:glycerol, choline chloride:glycerol and betaine:mannose at 5 and $50 \%(\mathrm{v} / \mathrm{v}) .{ }^{17}$ The results show that the replacement of acetone by the DES was possible for the synthesis of polycatechin. ${ }^{17}$ Nevertheless, and particularly taking into 
account the DES designer solvents feature, the use of DES as solvent media for enzymatic reactions with laccase must be more extensively studied aiming a deeper understanding on how DES and their compositions and concentrations in aqueous solutions can affect the enzymes activity and stability.

Chemical structures, hydrophobicity, polarity, viscosity, hydrogen bond basicity/acidity, among other properties, have been described to influence the enzymes activity and stability. ${ }^{18,19}$ However, the multitude of DES and their constituents and possible interactions with the enzymes amino acids turns the molecular-level understanding of their impact towards enzymatic activity a difficult task to be experimentally addressed. Aiming at solving this puzzle, computational methods can be applied. Among these, molecular docking is a fast and easy approach to identify possible preferential interactions between the amino acids of a target enzyme and the DES constituents, while providing additional values on the binding affinity of macromolecules (receptor) and small molecules. $^{20}$

In this work, the effect of DES as co-solvents aqueous solution on the laccase activity was evaluated at different temperatures and DES concentrations. Sixteen cholinium- or betaine-based DES were investigated by combining four HBAs, viz. choline chloride $(\mathrm{ChCl})$, choline dihydrogen phosphate (ChDHP), choline dihydrogen citrate (ChDHC) and betaine (Bet), with four HBDs compounds, namely ethylene glycol (EtG), glycerol (Gly), erytritol (Ery) and xylitol (Xyl). To better understand the molecular-level mechanisms responsible for the laccase activation or inactivation, molecular docking studies were carried out. 


\section{EXPERIMENTAL SECTION}

\section{Materials}

Laccase from Trametes versicolor (powder, light brown, $\geq 0.5{\mathrm{U} . \mathrm{mg}^{-1}}^{-1}$ ), betaine (Bet), choline dihydrogencitrate (ChDHC), citric acid, sodium phosphate dibasic, sodium phosphate monobasic, citric acid and 2,2'-azino-bis(3-ethylbenzathiazoline-6-sulfonic) acid (ABTS) were purchased from Sigma-Aldrich. Choline dihydrogenphosphate (ChDHP) were purchased from Iolitec. Ethylene glycol (EtG), and glycerol (Gly) were purchased from Fisher Chemical. Xylitol (Xyl) and Choline chloride $(\mathrm{ChCl})$ were purchased from Acros Organics. Meso - Erythritol (Ery) was purchased from Alfa Aesar. All compounds were of analytical grade and used without additional purification.

\section{Synthesis of DES}

The preparation of cholinium- and betaine-based DES was carried out by adding each hydrogen-bond acceptor (Bet, $\mathrm{ChCl}, \mathrm{ChDHC}$ or $\mathrm{ChDHP}$ ) to each hydrogen-bond donor (Ery, Gly, Xyl or EG) in glass vials at three molar ratios, namely 1:2, 1:1 and 2:1, resulting in the formation of liquid mixtures. Whenever necessary, the temperature was increased up to $80-100^{\circ} \mathrm{C}$ until the formation of a homogenous and clear liquid, particularly for Ery and Xyl based DES. All mixtures were stirred for $1 \mathrm{~h}$. After the synthesis of DES, three different concentrations $(10,25$ and $50 \mathrm{wt} \%)$ in aqueous solution were prepared for each DES and each molar ratio. The chemical structures of the investigated DES are given in Table 1. 
Table 1. Composition, chemical structures, nomenclature and molar ratios of the DESs.

HBA

HBD<smiles>C[N+](C)(C)CCO</smiles>

choline chloride $(\mathrm{ChCl})$
HO

Ethylene glycol (EtG)<smiles>C[N+](C)(C)CCO</smiles>

Choline dihydrogen citrate (ChDHC)<smiles>OCC(O)CO</smiles>

Glycerol (Gly)<smiles>C[N+](C)(C)CCO</smiles>

Choline dihydrogen phosphate (ChDHP)<smiles>OCC(O)C(O)CO</smiles>

Erythritol (Ery)<smiles>C[N+](C)(C)CC(=O)[O-]</smiles>

Betaine (Bet)<smiles>OCC(O)C(O)C(O)CO</smiles>

Xylitol (Xyl)

Molar ratio

\section{DES}

$2: 1 ; 1: 1 ; 1: 2$

$\mathrm{ChCl}$ :Ery; $\mathrm{ChCl}$ :Xyl

$2: 1 ; 1: 1 ; 1: 2$

ChDHP:EtG; ChDHP:Gly

ChDHP:Ery; ChDHP:Xyl

ChDHC:EtG; ChDHC:Gly

$2: 1 ; 1: 1 ; 1: 2$

ChDHC:Ery; ChDHC:Xyl

Bet:EtG; Bet:Gly

$2: 1 ; 1: 1 ; 1: 2$

Bet:Ery; Bet:Xyl 


\section{Laccase stability in DES}

Laccase stability was evaluated in a phosphate buffer $(50 \mathrm{mM}, \mathrm{pH} 7.0)$ aqueous solution containing DES at concentrations of $10 \%, 25 \%$ and $50 \%(\mathrm{wt} \%)$ at $25^{\circ} \mathrm{C}$. In all tests, the final laccase concentration was $0.25 \mathrm{~g} \mathrm{~L}^{-1}$. The mixture was incubated at room temperature for $10 \mathrm{~min}$ and then a sample was taken for laccase activity assay. Results presented in this work are the mean from at least three experiments.

The activity of laccase was assayed according to the method described by Ander and Messner. ${ }^{21}$ The enzymatic reaction was carried out at $25^{\circ} \mathrm{C}$ by adding $100 \mu \mathrm{L}$ of sample in $500 \mu \mathrm{L}$ of $0.4 \mathrm{mM}$ 2,2-azino-bis(3-ethylbenzthiazoline-6-sulfonic acid) (ABTS) and $1400 \mu \mathrm{L}$ of $50 \mathrm{mM}$ citrate $/ 100 \mathrm{mM}$ phosphate buffer at $\mathrm{pH} 4.5$. Since the ABTS oxidation is dependent on the $\mathrm{pH}$, the laccase activity method was carried out in acidic conditions. One unit (U) of laccase activity is defined as the amount of enzyme able to oxidize 1 $\mu$ mol of ABTS per minute. The oxidation of ABTS was monitored by the increase in absorbance measured in kinetic model of a UV-Vis spectrophotometer (Agilent 8453) at $420 \mathrm{~nm}\left(\varepsilon=36.000 \mathrm{M}^{-1} \mathrm{~cm}^{-1}\right)$. Laccase activities are presented in $\mathrm{U} \mathrm{L}^{-1}$ (for more details see the Supporting Information).

Laccase thermal stability was additionally evaluated by incubating the enzyme in absence and presence of DES aqueous solutions at $-80^{\circ} \mathrm{C}$ and $60^{\circ} \mathrm{C}$. Samples were collected at various intervals of time, and the residual activity was measured as described.

\section{Molecular Docking}

The interaction sites of laccase with all HBDs and HBAs that compose each DES were identified using the Auto-dock vina 1.1.2 program ${ }^{22}$. The crystal structure of laccase PDB: 1kya was used. Auto DockTools $(\mathrm{ADT})^{20}$ was used to prepare the enzyme input 
files by merging non-polar hydrogen atoms, adding partial charges and atom types. Ligand (HBDs and HBAs) 3D atomic coordinates were computed by Gaussian $03 \mathrm{w}$ and ligand rigid root was generated using AutoDockTools (ADT), setting all possible rotatable bonds defined as active by torsions. The grid center at the center of mass (x-, y, and z-axes, respectively) to cover the whole interaction surface of Laccase was $54 \AA \times$ $68 \AA \times 74 \AA$. The binding model that has the lowest binding free energy was searched out from 10 different conformers for each ligand (HBDs and HBAs).

\section{RESULTS AND DISCUSSION}

\section{Effect of DES nature and concentration on the laccase activity}

In the literature it has been demonstrating that enzymes are able to maintain and even enhance their activity and stability on DES-based media, thus acting as improved solvents or co-solvents over conventional organic solvents. ${ }^{8,15,16}$ Still, the catalytic behavior of laccase in DES is scarcely explored. Therefore, in this work, the activity of laccase was evaluated in presence of sixteen DES constituted by betaine or cholinium-based salts, as HBAs, and four polyols, namely ethylene glycol, glycerol, erythritol and xylitol, as HBDs. DES were prepared using different HBA:HBD molar ratios $(2: 1 ; 1: 1$ and 1:2) and at three different concentrations $(10,25$ and $50 \mathrm{wt} \%)$ in aqueous solution. The term DES aqueous solution was considered since data from the literature shows that DES maintain

their structure in aqueous solution, although dependent on the water content. ${ }^{23-26}$ The enzyme activity in the several DES aqueous solution was assessed by comparing with the ABTS oxidation catalyzed by laccase in phosphate buffer aqueous solutions (control, relative laccase activity of $100 \%$ ). Considering the different properties between aqueous buffer and DES solutions, the influence of the solvent viscosity was not evaluated. 
Besides the high viscosity of pure anhydrous DES when compared to water, Leija et al. ${ }^{27}$ proved that the viscosity of aqueous solutions of DES has no influence on enzyme activity. Other works have shown that the presence of water in DES results in a high viscosity decrease, attaining values close to the viscosity of water. ${ }^{26,28,29}$ The laccase activity at $25^{\circ} \mathrm{C}$ in presence $\mathrm{ChCl}$-based $\mathrm{DES}$ at different concentrations and compositions, including with the two DES constituents alone, are shown in Figure 1. Detailed results are given in the Supporting Information (Table S1 and Table S2). Overall, relative laccase activity values range from $19-98 \%$. From the results shown in Figure 1 it is clear that $\mathrm{ChCl}$ deactivates the enzyme; accordingly, all ChCl-based DES induce a decrease in the enzyme relative activity when compared to the control (phosphate buffer $50 \mathrm{mM}, \mathrm{pH} 7.0$ ), especially at the highest DES concentration of $50 \mathrm{wt} \%$ where the enzyme relative residual activity is below $40 \%$. This trend on the negative effect of $\mathrm{ChCl}$ is confirmed by the relative activity results in presence of aqueous solutions of ethylene glycol, glycerol, erythritol and xylitol at the same concentrations. As shown in Figure 1, the activity of laccase is particularly decreased in $\mathrm{ChCl}$ solutions, and higher in solutions of the individual polyols when compared to the respective DES. In summary, there is a negative effect of $\mathrm{ChCl}$ and a positive effect of polyols in the laccase activity, resulting in intermediate laccase activity values in presence of DES. The non-beneficial effect of $\mathrm{ChCl}$ towards the laccase activity is further supported by the results obtained when increasing the $\mathrm{ChCl}$ molar ratio from $1: 2$ to $2: 1$. These results show that the loss in laccase activity in these DES can be attributed to the presence of $\mathrm{Cl}^{-}$, and enzyme denaturating ion as reported in the literature, ${ }^{30}$ and/or due to an inhibitory effect of $\mathrm{ChCl}$ on the laccase activity by disrupting the intramolecular hydrogen bonds. ${ }^{16,31}$ The results obtained with polyols agree with literature, for instance, the activity of $\beta$-glucosidase was higher when 
polyols, namely ethylene glycol and propylene glycol, were used as constituent in $\mathrm{ChCl}$ based DES. ${ }^{32}$ The authors explain that a single constituent molecule may diffuse into the enzyme core causing its denaturation by disrupting intramolecular hydrogen bonds. ${ }^{32} \mathrm{On}$ the other hand, the formation of hydrogen bonds in DES may prevent its diffusion into the enzyme active centre. ${ }^{32}$ As DES are prepared by mixing a salt and a hydrogen-bond donor which may be dissociated upon dissolution into aqueous solution, it is reasonable to assume that the DES effects observed result from the combined action of the two DES components. ${ }^{8}$ A similar study using laccase from Escherichia coli BL21 and the glycerol:cholinium chloride DES (molar ratio 2:1) showed that no laccase activity was detected for DES concentrations above $30 \%(\mathrm{v} / \mathrm{v}){ }^{16}$
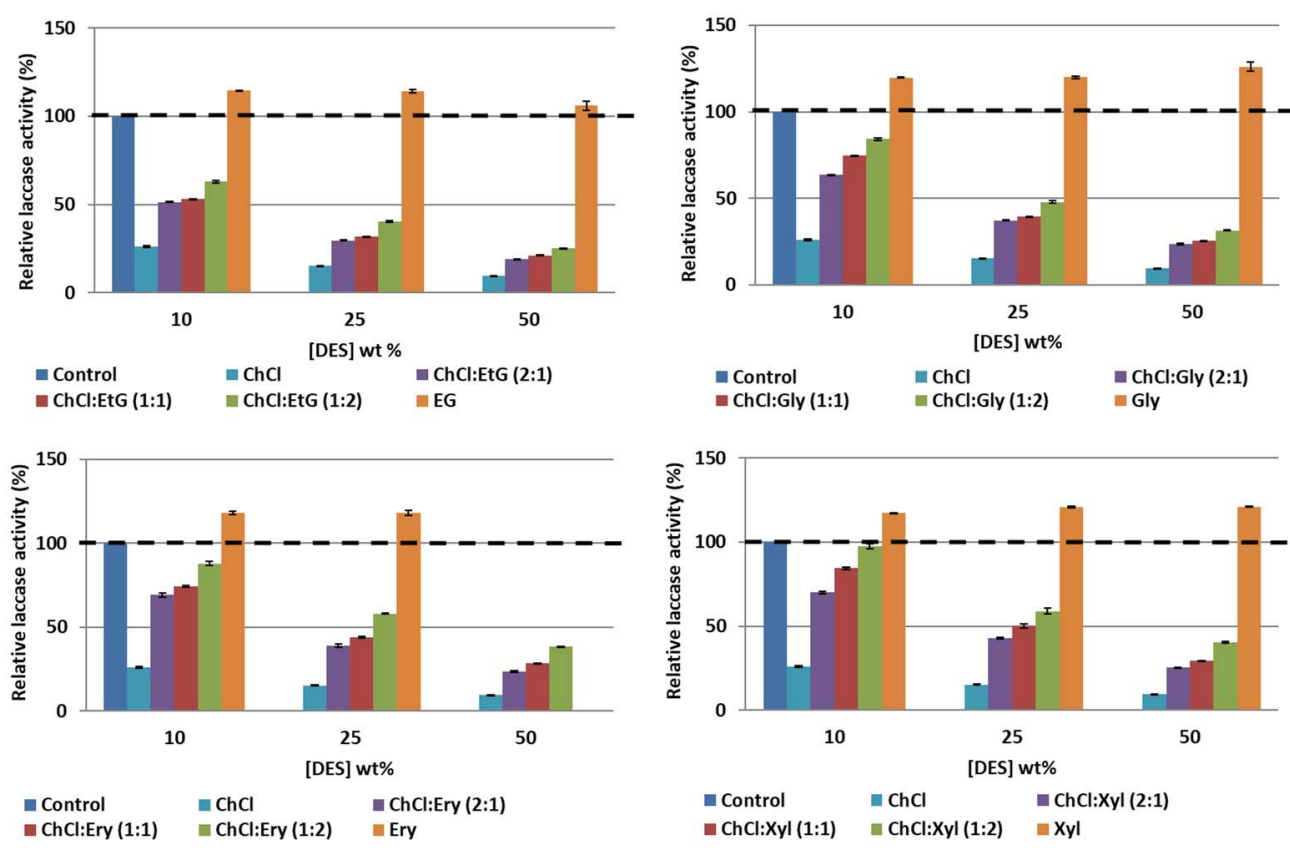

Figure 1. Relative activity (\%) of laccase in presence of ChCl-based DES using different HBA:HBD molar ratios $(2: 1 ; 1: 1$ and $1: 2)$ and of the individual constituents $(\mathrm{ChCl}, \mathrm{EtG}$, Gly, Ery and $\mathrm{Xyl})$ at different concentrations (10-50 wt\%) in aqueous solution (except for Ery at $50 \%$ wt since this compound was not soluble). Relative activity (\%) is 
addressed by comparison with the free-DES phosphate buffer $\mathrm{pH} 7.0$ and $50 \mathrm{mM}$ at $25^{\circ} \mathrm{C}$ (control, 100\%).

Due to the negative effects afforded by $\mathrm{ChCl}$ on the laccase activity, two additional cholinium-based salts were investigated, namely ChDHP and ChDHC. The results for ChDHP-based DES are depicted in Figure 2. Detailed results are given in the Supporting Information (Table S1 and Table S2). In general, and only by replacing the choliniumbased salt anion, an increase in the laccase activity was observed in respect to the control (relative laccase activity $>100 \%$ ). The only exceptions are seen for the DES ChDHP:EtG and for most DES at the highest concentration of $50 \mathrm{wt} \%$. A negative effect of pure ChDHP is only noticeable at the highest concentration $(50 \mathrm{wt} \%)$ as well. There is a noticeable activation of the enzyme by increasing the number of hydroxyl groups, with significant activity enhancements observed with the 1:2 DES based on Ery (four hydroxyl groups) and Xyl (five hydroxyl groups). In this set of result, a synergetic effect between the HBA and HBD on the enzyme activation is also observed, where DES present a better performance to improve the enzyme activity when compared to the isolated ChDHP and polyols. Overall, the ChDHP:Xyl (1:2) DES at 10 and $25 \mathrm{wt} \%$ lead to the best results with an increase in the laccase relative activity of about $70 \%$ when compared with the results obtained for the buffered control. 

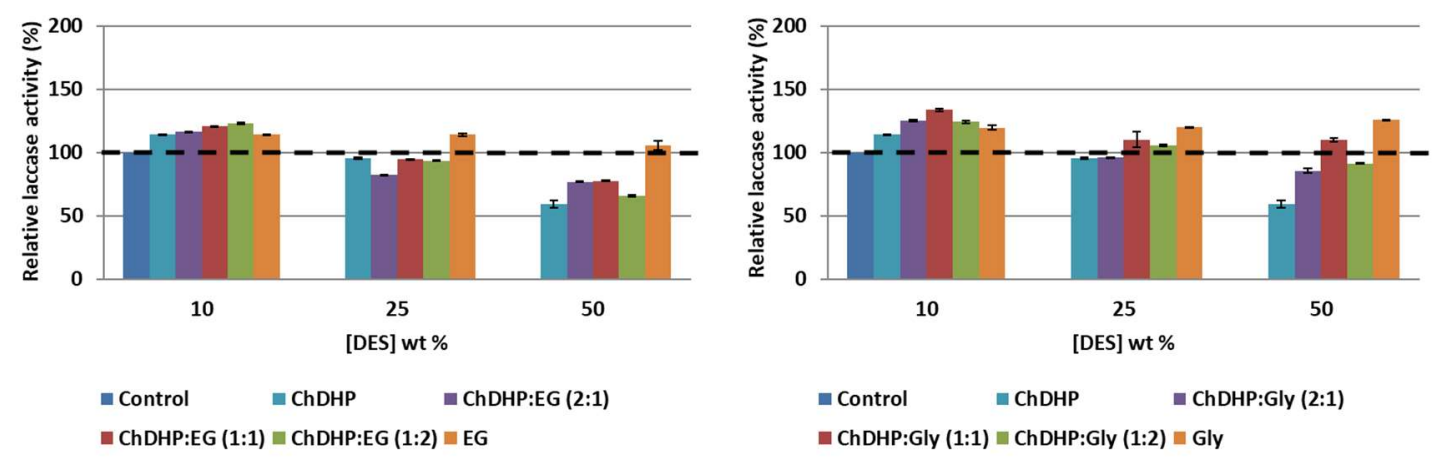

चChDHP:EG (1:1) $\approx$ ChDHP:EG (1:2) $\approx \mathrm{EG}$

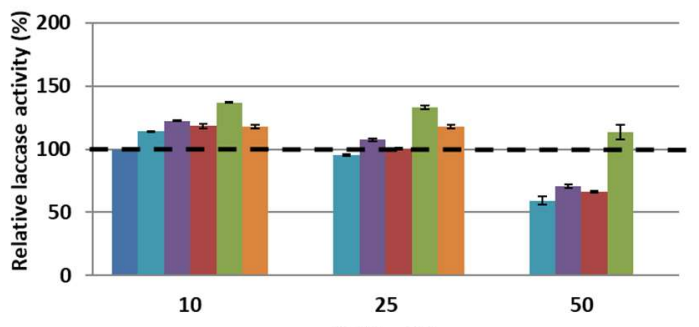
[DES] wt \%

a Control $\quad \square$ ChDHP $\quad$ ChDHP:Ery (2:1) ఐChDHP:Ery (1:1) $₫$ ChDHP:Ery (1:2) $\approx$ Ery

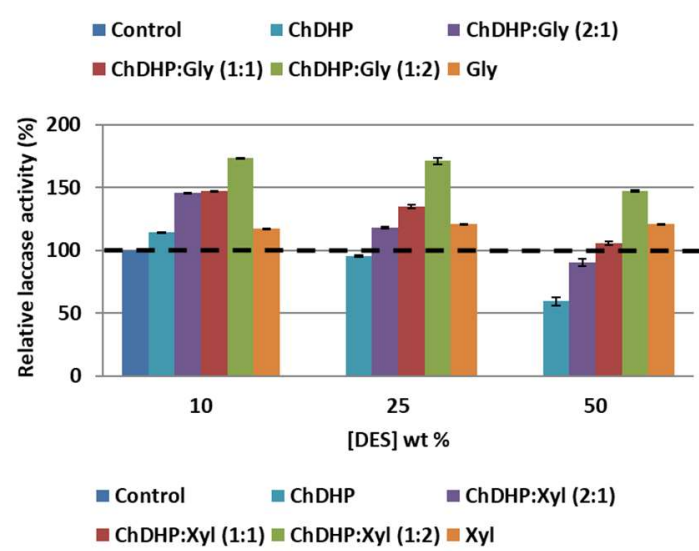

Figure 2. Relative activity (\%) of laccase in the presence of different concentrations (10$50 \%)$ of ChDHP-based DESs using different HBA:HBD molar ratios $(2: 1 ; 1: 1$ and $1: 2)$ and of the individual constituents $(\mathrm{ChCl}, \mathrm{EtG}, \mathrm{Gly}$, Ery and $\mathrm{Xyl})$ at different concentrations $(10-50 \mathrm{wt} \%$ ) in aqueous solution (except for Ery at $50 \%$ wt since this compound was not soluble). Relative activity (\%) is addressed by comparison with the free-DES phosphate buffer $\mathrm{pH} 7.0$ and $50 \mathrm{mM}$ at $25^{\circ} \mathrm{C}$ (control, 100\%).

The last cholinium-based DES investigated comprises ChDHC, which shows to be the most appropriate HBA amongst the several salts studied. The results presented in Figure 3 show that the laccase activity depends on the DES constituents, molar ratio and concentration. Detailed results are given in the Supporting Information (Table S1 and Table S2). Contrarily to what has been show before with $\mathrm{ChCl}$ and $\mathrm{ChDHP}, \mathrm{ChDHC}$ induces a relevant increase in the enzyme activity, which is even higher than the 
beneficial effect afforded by polyols. As observed with DES based on ChDHP, there is also a synergistic effect between the polyol and ChDHC since some DES have a superior performance to improve the enzymatic activity when compared to the respective constituents. Remarkable laccase activities ( $>170 \%)$ were obtained with DES constituted by $\mathrm{ChDHC}$ and Ery or $\mathrm{Xyl}$ at concentrations of 25 and $50 \mathrm{wt} \%$. In particular for ChDHC:Xyl, the enzyme activity increases with the DES concentration, being also dependent on the DES composition with an impact on the laccase activity following the trend: $2: 1>1: 1>1: 2$.

Comparing all ChDHC-based DES at a molar ratio $(2: 1)$ and at $50 \mathrm{wt} \%$, the increase in the enzyme activity again follows the order of the increasing number of hydroxyl groups in the polyol: ChDHC:EtG < ChDHC:Gly < ChDHC:Ery < ChDHC:Xyl. According to these results, it is clear that the laccase activity is dependent on the number of hydroxyl groups present in the polyols, and as observed for ChDHP-based DES. However, it should be remarked that the $\mathrm{ChDHC}$ also has 3 hydroxyl groups in the anion, thus being beneficial to improve the enzyme activity and explaining the best results achieved with DES formed by $\mathrm{ChDHC}$ and polyols. 

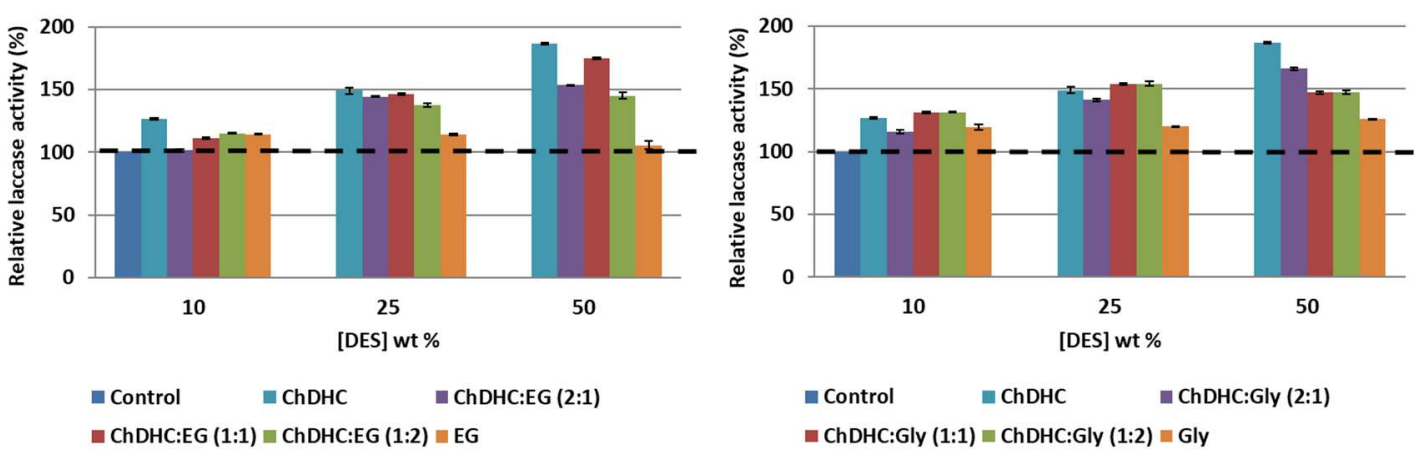

ChDHC:EG (1:1) 1 ChDHC:EG (1:2) $\equiv \mathrm{EG}$

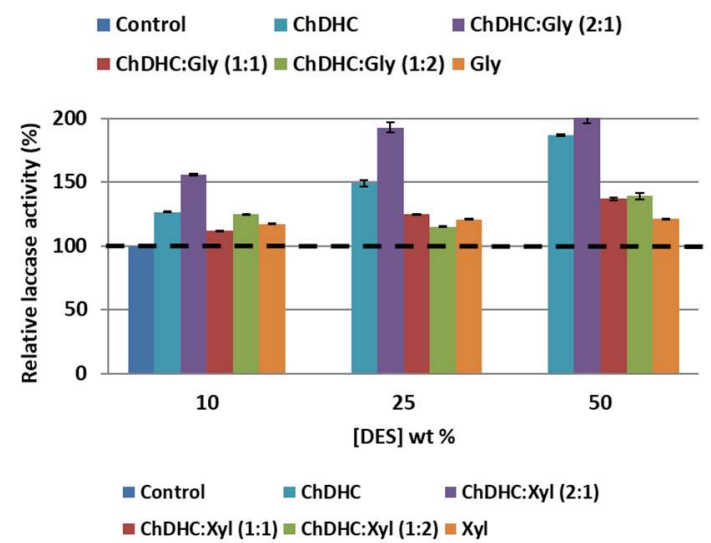

Figure 3. Relative activity (\%) of laccase in the presence of different concentrations (10$50 \%)$ of ChDHC-based DES using different HBA:HBD molar ratios $(2: 1 ; 1: 1$ and 1:2) and of the individual constituents $(\mathrm{ChCl}$, EtG, Gly, Ery and $\mathrm{Xyl})$ at different concentrations $(10-50 \mathrm{wt} \%$ ) in aqueous solution (except for Ery at $50 \%$ wt since this compound was not soluble). Relative activity (\%) is addressed by comparison with the free-DES phosphate buffer $\mathrm{pH} 7.0$ and $50 \mathrm{mM}$ at $25^{\circ} \mathrm{C}$ (control, $100 \%$ ).

It has been shown previously that betaine is able to preserve proteins against deactivation and aggregation. ${ }^{33}$ Furthermore, Khodaverdiana et al. ${ }^{16}$ provided encouraging results for improving laccase activity and stability using betaine-based natural DES. Therefore, the effect of betaine-based DES on the activity of laccase was investigated using the same polyols as HBD, allowing to address the effect of DES formed by cholinium-based salts versus betaine. Detailed results are given in the Supporting Information (Table S1 and Table S2). As presented in Figure 4, while this seems to hold true for the betaine aqueous 
solutions, the activity of laccase is similar for all solutions of betaine-based DES, with a maximum activity enhancement of $20 \%$ when compared to the buffer control results. Furthermore, betaine, unlike some of the cholinium-based salts studied before, does not lead to synergetic effects with polyols when combined to form DES. Overall, the best results were achieved with DES based on ChDHC and Ery or Xyl at concentrations of 25 and $50 \mathrm{wt} \%$, which seems to mainly derive from the increased number of hydroxyl groups in their chemical structures and their synergetic effect.
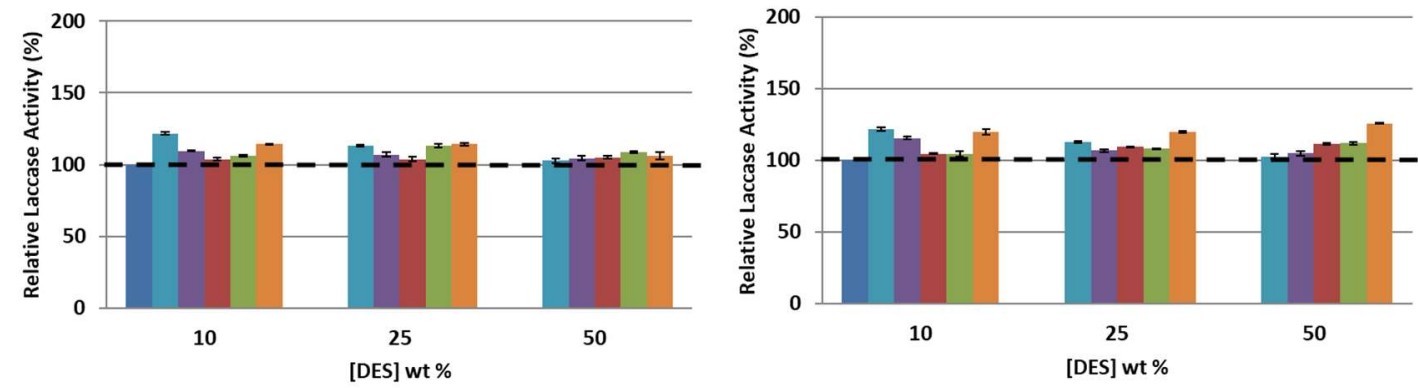

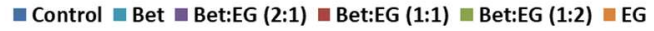
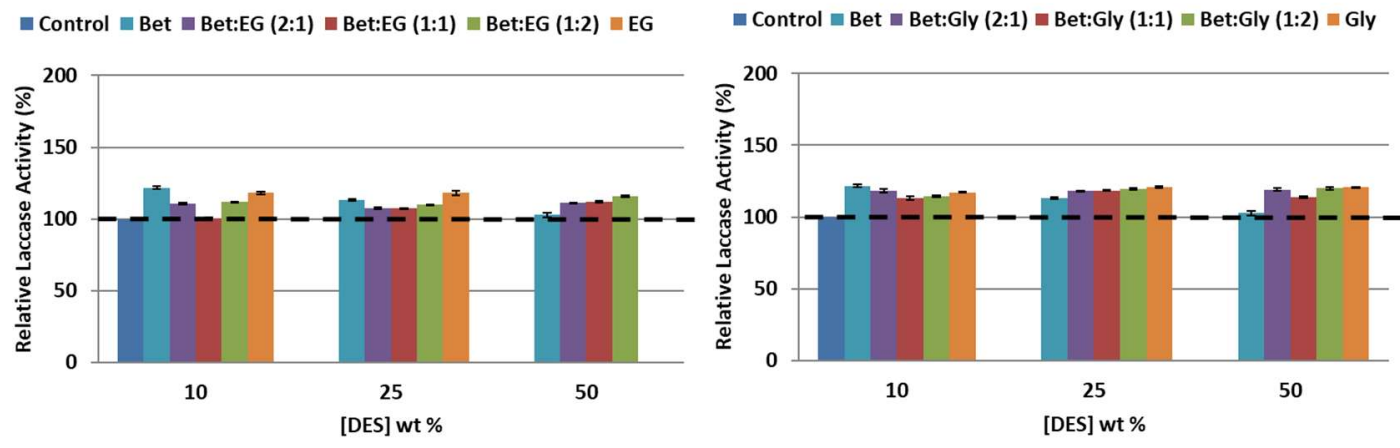

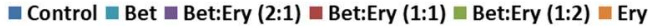

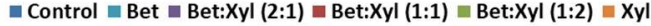

Figure 4. Relative activity of laccase in the presence of different concentrations (10$50 \%)$ of betaine-based DESs using different HBA:HBD molar ratios $(2: 1 ; 1: 1$ and $1: 2)$ and of the individual constituents ( $\mathrm{ChCl}$, EtG, Gly, Ery and $\mathrm{Xyl}$ ) at different concentrations $(10-50 \mathrm{wt} \%$ ) in aqueous solution (except for Ery at $50 \%$ wt since this compound was not soluble). Relative activity (\%) is addressed by comparison with the free-DES phosphate buffer $\mathrm{pH} 7.0$ and $50 \mathrm{mM}$ at $25^{\circ} \mathrm{C}$ (control, 100\%). 


\section{Molecular docking}

Aiming at better understanding the molecular-level effect of each HBD and HBA on the laccase activity, molecular docking calculations were carried out. The bind pose with lowest absolute value of affinity energy for laccase with all ions that constitute the HBAs in each DES $\left([\mathrm{Ch}]^{+}, \mathrm{Cl}^{-},[\mathrm{DHC}]^{-},[\mathrm{DHP}]^{-}\right.$and Betaine) are displayed in Figure 5, while the poses for the HBDs (EtG, Gly, Ery and Xyl) and all molecular interactions diagrams are shown in the Supporting Information. The best binding pose and docking affinity energies, interacting amino acids, type of interaction and distance of all HBDs and HBAs are provided in the Supporting Information (Table S4 and Table S5; Figures S1-S5.).

All polyols only establish hydrogen-bonding with enzyme amino acids, particularly with serine, alanine and histidine. Furthermore, the HBD-laccase absolute values of docking affinity energies increase in the order: ethylene glycol $<$ glycerol $<$ erythritol $<$ xylitol, in agreement with the increasing number of hydroxyl groups in the polyol and experimentally verified improvements on the laccase activity. Therefore, both by experimental results and by molecular docking it is clear that the laccase activity is dependent on the number of hydroxyl groups present in the polyols and their ability to hydrogen-bond with the enzyme amino acids. The absolute docking affinity energies between the ions that compose each HBA and laccase follow the rank: $[\mathrm{DHC}]^{-}>[\mathrm{DHP}]^{-}$ $>$ Betaine $>[\mathrm{Ch}]^{+}>\mathrm{Cl}^{-}$. This set is also in agreement with the experimental results on the laccase relative activity. As observed with polyols, the higher the interaction energy between each DES component or ion and the enzyme, the higher their beneficial effect towards the enzymatic activity. With the exception of $[\mathrm{Ch}]^{+}$and betaine that establish electrostatic interactions with aspartic acid amino acids, the remaining ions of each HDA 
only establish hydrogen-bonding with enzyme amino acids, namely with aspartic acid, tyrosine, histidine, arginine, alanine, serine, and glycine.

Histidine, cysteine and phenylalanine and four atoms of copper compose the catalytic cluster of laccase from Trametes versicolor. ${ }^{34}$ Therefore, the increase in the enzymatic activity could be a direct consequence of interactions established with histidine at the catalytic cluster. In fact, all HBDs have preference for binding to HIS111 in the laccase catalytic cluster. Accordingly, the most promising HBDAs constituents ([DHC] $]^{-},[\mathrm{DHP}]^{-}$ and Betaine) bind to HIS111 and HIS402. On the other hand, $[\mathrm{Ch}]^{+}$and $\mathrm{Cl}^{-}$display no affinity for HIS111 and HIS402, which may justify the low performance of $\mathrm{ChCl}$ to keep the laccase activity. Nevertheless, it should not be discarded the hypothesis that the interactions established between the DES components and the side chain amino acids may lead to conformational rearrangements of the enzyme, and as such, substrates can more easily access the enzyme active site.

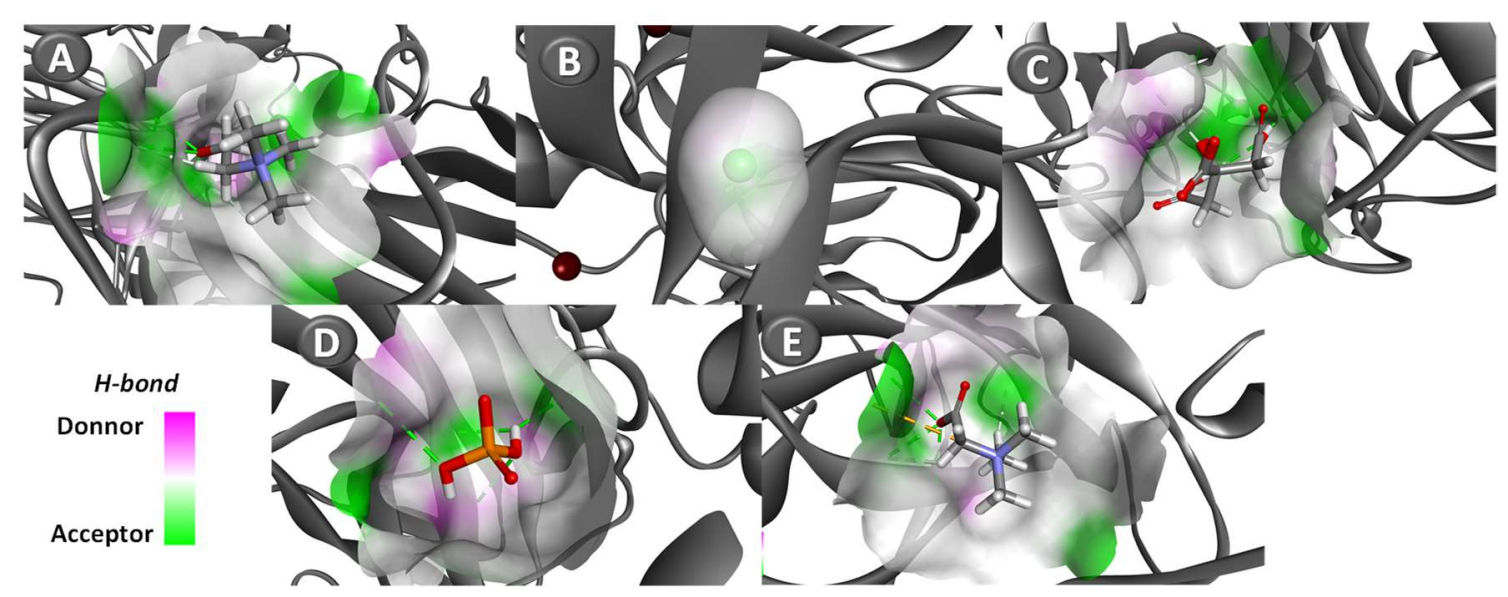

Figure 5. Docking pose with the lowest absolute value of affinity for laccase with: (A) $[\mathrm{Ch}]^{+},(\mathrm{B}) \mathrm{Cl}^{-},(\mathrm{C})[\mathrm{DHC}]^{-},(\mathrm{D})[\mathrm{DHP}]^{-}$and (E) Betaine. 


\section{Thermal stability of laccase in DES}

Based on the results presented above, the thermal stability of laccase was evaluated with three DES, namely ChDHP:Xyl (1:2) at $10 \mathrm{wt} \%$, ChDHP:Xyl (1:2) at $25 \mathrm{wt} \%$, and ChDHC:Xyl (2:1) at $25 \mathrm{wt} \%$, by incubating the enzyme under two extreme temperatures, viz. $60^{\circ} \mathrm{C}$ and $-80^{\circ} \mathrm{C}$, up to 20 days. These DES and molar ratio were selected to address the DES constituents effect and their molar ratio. The respective results are shown in Figure 6. Detailed results are given in the Supporting Information (Table S3). As before, the relative activity was determined in respect to the control (initial laccase activity in buffer solution at the same conditions) considered as 100\%. Although these DES are advantageous to keep the enzymatic activity when compared to the control at $60^{\circ} \mathrm{C}$, after incubation for two days there is decrease in the activity of laccase with both the buffered control solution and the investigated DES (Figure 6 A). After two days of incubation at $60^{\circ} \mathrm{C}$, only $5-10 \%$ of enzyme activity was detected in presence of ChDHP:Xyl (1:2) at 10 wt $\%$ and ChDHP:Xyl (1:2) and no activity for ChDHC:Xyl (2:1) at $25 \mathrm{wt} \%$. After six days no laccase activity was observed with none of the investigated solvents. On the other hand, a remarkable storage thermal stability was obtained at $-80^{\circ} \mathrm{C}$ in presence of these DES, as shown in Figure 6 B, with relative activity values ranging between 130 and 200\% up to 20 days of storage. The enhancement of the laccase activity, e.g. up to nine days of incubation, follows the order: ChDHC:Xyl (2:1) at $25 \mathrm{wt} \%>$ ChDHP:Xyl (1:2) at 25 $\mathrm{wt} \%>\mathrm{ChDHP}: \mathrm{Xyl}(1: 2)$ at $10 \mathrm{wt} \%>\operatorname{control}($ no DES). After this period and up to twenty days of incubation, the remaining activity of laccase decreases, but still remains above the control. As observed with the results at $25^{\circ} \mathrm{C}$, the DES ChDHC:Xyl (2:1) at $25 \mathrm{wt} \%$ is the most promising to storage and keep the laccase activity along time. This behaviour of DES on stabilizing biomolecules at very low temperatures was already applied for the 
development of new cryoprotective agents. ${ }^{35}$ For instance, natural deep eutectic systems (NADES) based on trehalose and glycerol were used as alternative nontoxic cryoprotective agents for a model cell line L929. ${ }^{36}$ The authors concluded that aqueous solutions of NADES have a robust effect on the water crystallization/freezing and melting process, being able to reduce the number of ice crystals and hence ice crystal damage in cells. $^{36}$
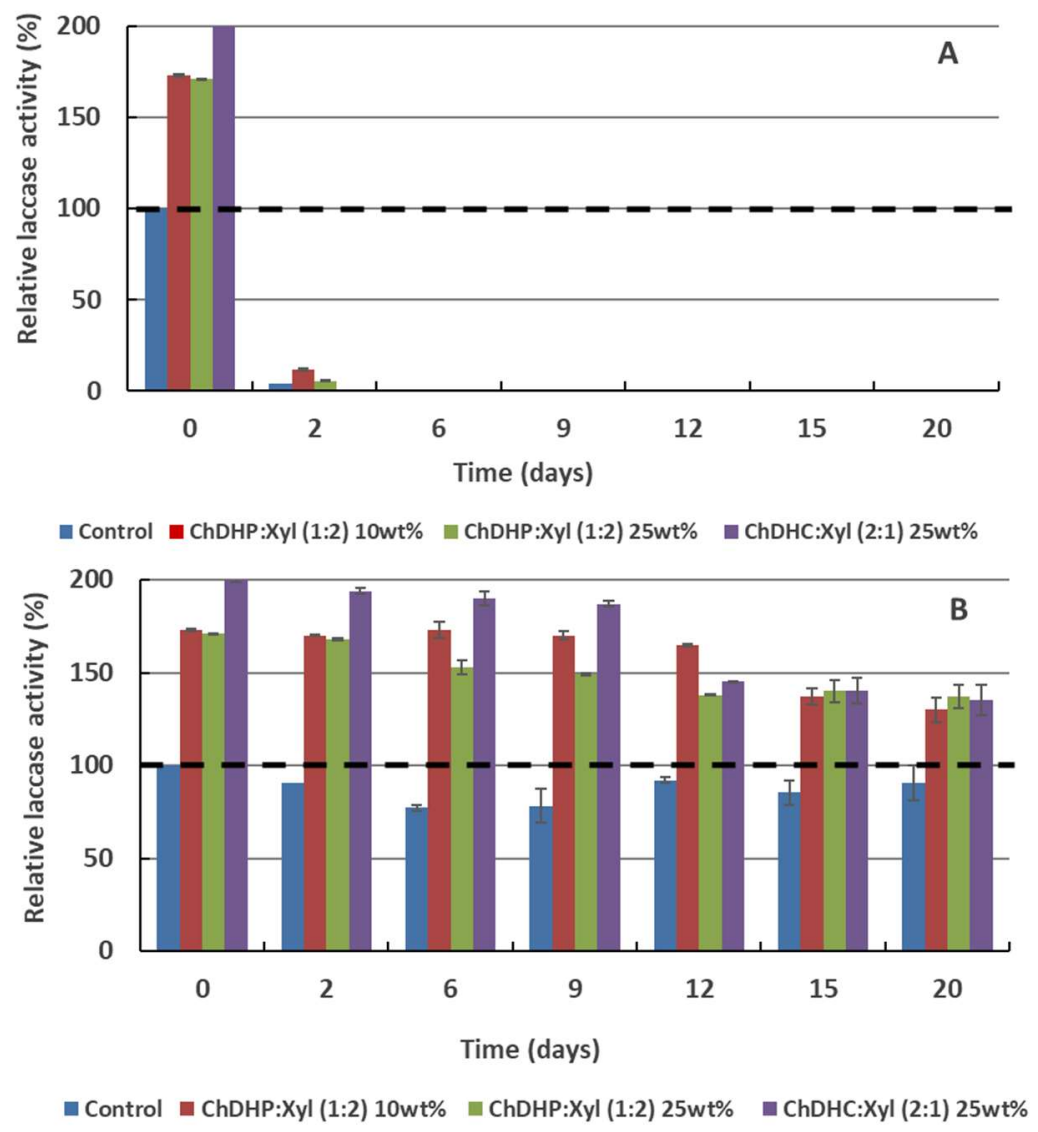

Figure 6. Relative activity of laccase in presence of ChDHC:Xyl (2:1) at $25 \mathrm{wt} \%$, ChDHP:Xyl (1:2) at $25 \mathrm{wt} \%$ and $\mathrm{ChDHP}: \mathrm{Xyl}(1: 2)$ at $10 \mathrm{wt} \%$ at $60^{\circ} \mathrm{C}(\mathrm{A})$ and $-80^{\circ} \mathrm{C}(\mathrm{B})$. Relative activity (\%) is addressed by comparison with the free-DES phosphate buffer 50 mM, pH 7.0 (control, 100\%). 


\section{CONCLUSIONS}

The catalytic activity of laccase in presence of sixteen cholinium- and betaine-based DES and of the respective constituents, and at different molar ratio, concentration and temperature was investigated. Although all polyols investigated are favorable to increase the laccase activity, $\mathrm{ChCl}$ and $\mathrm{ChCl}$-based $\mathrm{DES}$ lead to a decrease in the enzyme activity. However, the replacement of the chloride anion in the cholinium salts leads to significant improvements in the laccase activity. In particular, the DES ChDHP:Xyl and all ChDHCbased DES are improved co-solvents and significantly enhance the activity of laccase in oxidative reactions. Moreover, the enzyme activity in these DES aqueous solutions is higher than in aqueous solution of its individual components and in the buffer control, demonstrating a synergetic effect. Overall, enhancements in the relative laccase activity up to $200 \%$ at $25^{\circ} \mathrm{C}$ were observed. Furthermore, it was demonstrated that aqueous solutions of DES are better storage media than the respective buffer control at $-80^{\circ} \mathrm{C}$, where the relative activity of laccase is kept up to $135 \%$ for 20 days.

By molecular docking studies, it was demonstrated that all polyols and HBD components mainly establish hydrogen-bonding with the enzyme amino acids, with the exception of $[\mathrm{Ch}]^{+}$and betaine that also establish electrostatic interactions with aspartic acid amino acids. Furthermore, the higher the HBD- or HBA-laccase absolute values of docking affinity energies the higher the activity of laccase, which is dependent on the number of hydroxyl groups present in the DES components and their ability to hydrogen-bond with the enzyme amino acids. Although interactions established between the DES components and the side chain amino acids may lead to conformational rearrangements of the enzyme leaving its active site more accessible to substrates, the overall results indicate that the 
increase in the enzymatic activity could be a direct consequence of interactions established with histidine at the catalytic cluster.

The present work demonstrates that the type of DES, molar ratio and concentration are fundamental issues to improve laccase oxidation reactions. These results suggest that by choosing DES appropriately they can act as remarkable solvents or co-solvents to improve biocatalysis performance. The results provided reveal the potential perspectives of application of these benign solvents or co-solvents to multiple oxidative biotransformations employing non-aqueous soluble laccase substrates, such as lignin (biodelignification and biobleaching of paper pulp), dyes (wastewater decolorization), phenolic compounds (bioremediation) and organic substrates used by pharmaceutical and food industries.

\section{AUTHOR INFORMATION}

\section{Corresponding Author}

*E-mail address: aptavares@ua.pt

\section{Author Contributions}

All authors have given approval to the final version of the manuscript.

\section{ACKNOWLEDGEMENTS}

This work was developed within the scope of the project CICECO-Aveiro Institute of Materials, FCT Ref. UID/CTM/50011/2019, financed by national funds through the FCT/MCTES and within the project POCI-01-0145-FEDER-031268 - funded by FEDER, through COMPETE2020 - Programa Operacional Competitividade e Internacionalização (POCI), and by national funds (OE), through FCT/MCTES. Mariah L. Toledo acknowledges University of São Paulo (Mobilidade internacional de duração 
especial). Ana P. M. Tavares acknowledges FCT for the Investigator Programme (IF/01634/2015). Matheus M. Pereira acknowledges the PhD grant (2740-13-3) and financial support from Coordenação de Aperfeiçoamento de Pessoal de Nível Superior Capes. João P. A. Silva acknowledges the support of FAPESP (Fundação de Amparo à Pesquisa do Estado de São Paulo-Process Number 2016/06683-0).

This article was presented at the 13th International Chemical and Biological Engineering Conference (CHEMPOR 2018). The authors acknowledge the Scientific and Organizing Committees of the Conference for the opportunity to present this work.

\begin{abstract}
ABBREVIATIONS
ILs, ionic liquids; DES, deep eutectic solvents; HBD, hydrogen bond donor; HBA, hydrogen bond acceptor; $\mathrm{ChCl}$, choline chloride; $\mathrm{ChDHP}$, choline dihydrogen phosphate; ChDHC, choline dihydrogen citrate; Bet, betaine; EtG, ethylene glycol; Gly, glycerol; Ery, erytritol; Xyl, xylitol; ABTS, 2,2'-azino-bis(3-ethylbenzathiazoline-6-sulfonic) acid.
\end{abstract}

\title{
Supporting information
}

Detailed method for laccase activity tests; relative laccase activity data in different DES at different concentrations and molar ratio; docking affinity energy data and interacting amino acids residues predicted by AutoDock vinna for Laccase and the DES HBAs and for laccase and DES HBDs; docking pose image with the lowest absolute value of affinity for laccase with: (A) ethylene glycol, (B) glycerol, (C) erythritol and (D) xylitol; molecular interaction diagrams of glycerol and xylitol and amino acids residues of laccase; molecular interaction diagrams of erythritol and ethylene glycol and amino acids residues of laccase; molecular interaction diagrams of $[\mathrm{Ch}]^{+}$and betaine and amino acids 
residues of laccase; molecular interaction diagrams of $[\mathrm{DHC}]^{-}$and $[\mathrm{DHP}]^{-}$and amino acids residues of laccase.

\section{REFERENCES}

1. Xu P, Zheng G-W, Du P-X, Zong M-H, Lou W-Y. Whole-Cell Biocatalytic Processes with Ionic Liquids. ACS Sustain Chem Eng. 2016;4(2):371-386. doi:10.1021/acssuschemeng.5b00965

2. Yang T-X, Zhao L-Q, Wang J, et al. Improving Whole-Cell Biocatalysis by Addition of Deep Eutectic Solvents and Natural Deep Eutectic Solvents. ACS Sustain Chem Eng. 2017;5(7):5713-5722. doi:10.1021/acssuschemeng.7b00285

3. Doukyu N, Ogino H. Organic solvent-tolerant enzymes. Biochem Eng J. 2010;48(3):270-282. doi:10.1016/J.BEJ.2009.09.009

4. Tavares P. M. A, Rodriguez O, Macedo A. E. New Generations of Ionic Liquids Applied to Enzymatic Biocatalysis. In: Ionic Liquids - New Aspects for the Future. InTech; 2013. doi:10.5772/51897

5. Couling DJ, Bernot RJ, Docherty KM, Dixon JK, Maginn EJ. Assessing the factors responsible for ionic liquid toxicity to aquatic organisms via quantitative structureproperty relationship modeling. Green Chem. 2006;8(1):82-90. doi:10.1039/B511333D

6. $\quad$ Engel P, Mladenov R, Wulfhorst H, Jäger G, Spiess AC. Point by point analysis: how ionic liquid affects the enzymatic hydrolysis of native and modified cellulose. Green Chem. 2010;12(11):1959-1966. doi:10.1039/C0GC00135J

7. Zhang Q, De Oliveira Vigier K, Royer S, Jérôme F. Deep eutectic solvents: syntheses, properties and applications. Chem Soc Rev. 2012;41(21):7108. 
doi:10.1039/c2cs35178a

8. Wu B-P, Wen Q, Xu H, Yang Z. Insights into the impact of deep eutectic solvents on horseradish peroxidase: Activity, stability and structure. J Mol Catal B Enzym. 2014;101:101-107. doi:10.1016/J.MOLCATB.2014.01.001

9. Smith EL, Abbott AP, Ryder KS. Deep Eutectic Solvents (DESs) and Their Applications. Chem Rev. 2014;114(21):11060-11082. doi:10.1021/cr300162p

10. Ducros V, Brzozowski AM, Wilson KS, et al. Crystal structure of the type-2 Cu depleted laccase from Coprinus dnereus at $2.2 \AA$ resolution. Nat Struct Biol. 1998;5(4):310-316. doi:10.1038/nsb0498-310

11. Puente-Santiago AR, Rodríguez-Padrón D, Quan X, et al. Unprecedented Wiring Efficiency of Sulfonated Graphitic Carbon Nitride Materials: Toward HighPerformance Amperometric Recombinant CotA Laccase Biosensors. ACS Sustain Chem Eng. 2019;7(1):1474-1484. doi:10.1021/acssuschemeng.8b05107

12. Cannatelli MD, Ragauskas AJ. Two Decades of Laccases: Advancing Sustainability in the Chemical Industry. Chem Rec. 2017;17(1):122-140. doi:10.1002/tcr.201600033

13. d'Acunzo F, Barreca AM, Galli C. Determination of the activity of laccase, and mediated oxidation of a lignin model compound, in aqueous-organic mixed solvents. $\quad J \quad$ Mol Catal $B \quad$ Enzym. 2004;31(1-3):25-30. doi:10.1016/J.MOLCATB.2004.07.001

14. Gotor-Fernández V, Paul CE. Deep eutectic solvents for redox biocatalysis. $J$ Biotechnol. 2019;293:24-35. doi:10.1016/J.JBIOTEC.2018.12.018

15. Gorke JT, Srienc F, Kazlauskas RJ. Hydrolase-catalyzed biotransformations in deep eutectic solvents. Chem Commun. 2008;(10):1235. doi:10.1039/b716317g 
16. Khodaverdian S, Dabirmanesh B, Heydari A, Dashtban-moghadam E, Khajeh K, Ghazi F. Activity, stability and structure of laccase in betaine based natural deep eutectic solvents. Int $J$ Biol Macromol. 2018;107:2574-2579. doi:10.1016/J.IJBIOMAC.2017.10.144

17. Ezgi Ünlü A, Prasad B, Anavekar K, Bubenheim P, Liese A. Investigation of a green process for the polymerization of catechin. Prep Biochem Biotechnol. 2017;47(9):918-924. doi:10.1080/10826068.2017.1365241

18. Gao W-W, Zhang F-X, Zhang G-X, Zhou C-H. Key factors affecting the activity and stability of enzymes in ionic liquids and novel applications in biocatalysis. Biochem Eng J. 2015;99:67-84. doi:10.1016/J.BEJ.2015.03.005

19. Zhao H. Protein stabilization and enzyme activation in ionic liquids: specific ion effects. J Chem Technol Biotechnol. 2016;91(1):25-50. doi:10.1002/jctb.4837

20. Morris GM, Huey R, Lindstrom W, et al. AutoDock4 and AutoDockTools4: Automated docking with selective receptor flexibility. J Comput Chem. 2009;30(16):2785-2791. doi:10.1002/jcc.21256

21. Ander P, Messner K. Oxidation of 1-hydroxybenzotriazole by laccase and lignin peroxidase. Biotechnol Tech. 1998;12(3):191-195. doi:10.1023/A:1008813206178

22. Trott O, Olson A. AutoDock Vina: improving the speed and accuracy of docking with a new scoring function, efficient optimization and multithreading. $J$ Comput Chem. 2010;31(2):455-461. doi:10.1002/jcc.21334.AutoDock

23. Hammond OS, Bowron DT, Edler KJ. The Effect of Water upon Deep Eutectic Solvent Nanostructure: An Unusual Transition from Ionic Mixture to Aqueous Solution. Angew Chemie Int Ed. 2017;56(33):9782-9785. 
doi:10.1002/anie.201702486

24. Hammond OS, Bowron DT, Jackson AJ, et al. Resilience of Malic Acid Natural Deep Eutectic Solvent Nanostructure to Solidification and Hydration. J Phys Chem B. 2017;121(31):7473-7483. doi:10.1021/acs.jpcb.7b05454

25. Hammond OS, Edler KJ, Bowron DT, Torrente-Murciano L. Deep eutecticsolvothermal synthesis of nanostructured ceria. Nat Commun. 2017;8:14150. https://doi.org/10.1038/ncomms14150.

26. Dai Y, Witkamp G-J, Verpoorte R, Choi YH. Tailoring properties of natural deep eutectic solvents with water to facilitate their applications. Food Chem. 2015;187:14-19. doi:10.1016/J.FOODCHEM.2015.03.123

27. Sánchez-Leija RJ, Torres-Lubián JR, Reséndiz-Rubio A, Luna-Bárcenas G, MotaMorales JD. Enzyme-mediated free radical polymerization of acrylamide in deep eutectic solvents. RSC Adv. 2016;6(16):13072-13079. doi:10.1039/C5RA27468K

28. Zdanowicz M, Wilpiszewska K, Spychaj T. Deep eutectic solvents for polysaccharides processing. A review. Carbohydr Polym. 2018;200:361-380. doi:10.1016/J.CARBPOL.2018.07.078

29. Dai Y, van Spronsen J, Witkamp G-J, Verpoorte R, Choi YH. Natural deep eutectic solvents as new potential media for green technology. Anal Chim Acta. 2013;766:61-68. doi:10.1016/J.ACA.2012.12.019

30. Zhao H, Baker GA, Holmes S. Protease activation in glycerol-based deep eutectic solvents. $\quad J \quad$ Mol Catal B Enzym. 2011;72(3-4):163-167. doi:10.1016/j.molcatb.2011.05.015

31. Johannes C, Majcherczyk A. Laccase activity tests and laccase inhibitors. $J$ Biotechnol. 2000;78(2):193-199. doi:10.1016/S0168-1656(00)00208-X 
32. $\mathrm{Xu} \mathrm{W-J,} \mathrm{Huang} \mathrm{Y-K,} \mathrm{Li} \mathrm{F,} \mathrm{et} \mathrm{al.} \mathrm{Improving} \beta$-glucosidase biocatalysis with deep eutectic solvents based on choline chloride. Biochem Eng J. 2018;138:37-46. doi:10.1016/J.BEJ.2018.07.002

33. Ghazi A, Demont-Caulet N, Richarme G, Caldas T. Thermoprotection by glycine betaine and choline. Microbiology. 1999;145(9):2543-2548. doi:10.1099/00221287-145-9-2543

34. Piontek K, Antorini M, Choinowski T. Crystal Structure of a Laccase from the Fungus Trametes versicolor at 1.90- $\AA$ Resolution Containing a Full Complement of Coppers. J Biol Chem. 2002;277(40):37663-37669. doi:10.1074/jbc.M204571200

35. Gertrudes A, Craveiro R, Eltayari Z, Reis RL, Paiva A, Duarte ARC. How Do Animals Survive Extreme Temperature Amplitudes? The Role of Natural Deep Eutectic Solvents. ACS Sustain Chem Eng. 2017;5(11):9542-9553. doi:10.1021/acssuschemeng.7b01707

36. Castro VIB, Craveiro R, Silva JM, Reis RL, Paiva A, C. Duarte AR. Natural deep eutectic systems as alternative nontoxic cryoprotective agents. Cryobiology. 2018;83:15-26. doi:10.1016/J.CRYOBIOL.2018.06.010 


\section{SYNOPSIS}

Improved sustainable laccase biocatalysis in deep eutectic solvents

\section{ABSTRACT GRAPHIC}

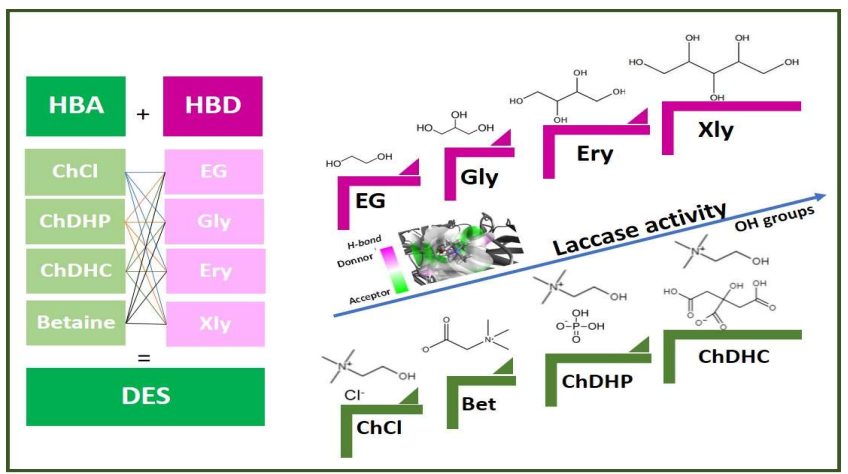

